\title{
Benign paroxysmal positional vertigo and osteoporosis
}

\author{
Shih-Wei Lai ${ }^{1,2}$ (1) \\ Received: 8 December 2018 / Accepted: 12 December 2018 / Published online: 15 December 2018 \\ (c) Springer-Verlag GmbH Germany, part of Springer Nature 2018
}

One case-control study by Choi et al. published in Eur Arch Otorhinolaryngol demonstrated that patients with benign paroxysmal positional vertigo were 1.29 times more likely to have osteoporosis than those patients without benign paroxysmal positional vertigo (adjusted odds ratio 1.29; 95\% confidence interval 1.23-1.35) [1]. Some of my ideas are shared with the readers. First, Table 1 in Choi et al. study demonstrated that the prevalence rates of osteoporosis were $28.16 \%(3797 / 13,484)$ in the benign paroxysmal positional vertigo group and $23.47 \%(12,657 / 53,936)$ in the control group [1]. These data are different from the description in result section. Second, Choi et al. study was a case-control study and the outcome was benign paroxysmal positional vertigo. That is, the authors selected cases with benign paroxysmal positional vertigo and controls without benign paroxysmal positional vertigo. Then, cases and controls were compared to retrospectively examine whether or not they had osteoporosis before benign paroxysmal positional vertigo. A causal relationship cannot be established by a case-control study. However, Choi et al. findings can be supported by a cohort study in Taiwan showing that patients with osteoporosis were 1.82 times more likely to develop benign paroxysmal positional vertigo than those patients without osteoporosis (adjusted hazard ratio 1.82; 95\% confidence interval 1.34-2.48) [2]. One case-control study in Japan also demonstrated that the recurrence rate of benign paroxysmal positional vertigo was significantly higher in patients with osteoporosis than those patients with normal bone mineral density (56.3\% versus $16.2 \%$ ) [3]. Finally, the association between benign paroxysmal positional vertigo and osteoporosis is a new topic, but related studies are few. More real-world data are necessary to examine this kind of association. I appreciate Choi et al. great efforts to provide the up-dated study on this topic.

\section{Compliance with ethical standards}

Conflict of interest The author discloses no conflicts of interest.

\section{References}

1. Choi HG, Lee JK, Kong IG, Lim H, Kim SY (2018) Osteoporosis increases the risk of benign paroxysmal positional vertigo: a nested case-control study using a national sample cohort. Eur Arch Otorhinolaryngol. https://doi.org/10.1007/s00405-018-5230-y

2. Chan KC, Tsai YT, Yang YH, Chen PC, Chang PH (2017) Osteoporosis is associated with increased risk for benign paroxysmal positional vertigo: a nationwide population-based study. Arch Osteoporos 12(1):017-0403

3. Yamanaka T, Shirota S, Sawai Y, Murai T, Fujita N, Hosoi H (2013) Osteoporosis as a risk factor for the recurrence of benign paroxysmal positional vertigo. Laryngoscope 123(11):2813-2816
Shih-Wei Lai

wei@mail.cmuh.org.tw

1 College of Medicine, China Medical University, Taichung 404, Taiwan

2 Department of Family Medicine, China Medical University Hospital, No 2, Yu-De Road, Taichung 404, Taiwan 